\title{
Modeling a 3G Power Control Algorithm in the MAC Layer for Multimedia Support
}

\author{
Ulises Pineda ${ }^{1}$, César Vargas ${ }^{2}$, Jesús Acosta-Elías ${ }^{1}$, J.M. Luna ${ }^{1}$, \\ Gustavo Pérez ${ }^{1}$, and Enrique Stevens ${ }^{1}$ \\ 1 Facultad de Ciencias, Universidad Autonoma de \\ San Luis Potosí, Av. Salvador Nava s/n, Zona Universitaria, \\ San Luis Potosí, S.L.P., 78290, México. \\ Tel: +52 (444) 8262316 , Fax: +52(444) 8262321 \\ \{u_pineda, estevens, jacosta, mlr\}@fc.uaslp.mx, \\ http://www.fc.uaslp.mx \\ 2 ITESM-CET, Monterrey, N.L., 64849, Mexico, \\ cvargas@itesm.mx
}

\begin{abstract}
Modern Third Generation Wireless Networks demand more and more resources in order to satisfy customers' needs. And these resources can only be provided by a good Power Control. However, power control needs an algorithm to work at the margin of the Quality of Service $(Q o S)$ requirements. This work proposes a power control algorithm modeled under probabilistic criteria. By means of applying a Markovian model to a MAC Protocol (power control algorithm), to optimize the power assignment to each user in the system. This protocol is highly interrelated to the power control functionality to extract the maximum capacity and flexibility out of the WCDMA scheme.
\end{abstract}

\section{Introduction}

Recently, extensive investigations have been carried out into the application of a Code Division Multiple Access (CDMA) as an air interface multiple access scheme for IMT-2000 (International Mobile Telecommunications System) - 2000 / UMTS (Universal Mobile Telecommunication System). CDMA is the technology for the third generation wireless personal communication systems. 1]

Power control is the single most important system requirement for CDMA based wireless networks systems. In the absence of power control the effect of near/far phenomena is dominant, and the capacity of the CDMA mobile system is very low. Power control allows users to share system resources equally between themselves. Besides furthermore, with a proper power control it is possible to lower transmitting power of the mobiles and prolong the battery life.

With this in mind, we will improve a MAC power control algorithm previously proposed in [2] and enhance its capabilities for an specific application: two services (voice and multimedia) with different rates in a $3 \mathrm{G}$ wireless network. 


\section{Model Description}

Based on a Markovian process, the On-Off model or Eng-Set distribution offers an accurate information about transmission activity or inactivity of users. Using this and adding it to a practical MAC protocol algorithm for power control, we can determine with accuracy the activity of users transmitting, and to establish the power vector $P_{R}$. The power vector $P_{R}$ describes the total power transmitted.

\subsection{Proposed Model}

Taking the multimedia MAC protocol proposed in [2], and thinking about to enhance their capabilities, we extend the protocol to consider more than one service. The next step is to establish a power control vector determined by an On-Off model with the purpose of knowing accurately how many users are in activity, and give them sufficient power without causing interference to the rest and keeping the $Q o S$ requirements.

Determination of the Number of Users. The number of packet transmission that could be supported in the next frame is calculated on a frame by frame basis to ensure the different Bit Error Rate (BER) requirements of all type of users. The general case for the power vector $P_{R}$ is defined as

$$
\begin{aligned}
\min \left\{P_{R}=\right. & \sum_{n_{1}=0}^{N_{1}} \sum_{n_{2}=0}^{N_{2}} \cdots \sum_{n_{k}=0}^{N_{k}} P_{n_{1}, n_{2}, \ldots, n_{k}} \cdot \\
& \left.\left(\sum_{j_{1}=1}^{n_{1}} P_{1, j_{1}}+\sum_{j_{2}=1}^{n_{2}} P_{2, j_{2}}+\cdots+\sum_{j_{k}=1}^{n_{k}} P_{k, j_{k}}\right)\right\},
\end{aligned}
$$

where the services are subject to the $Q o S$ constraints established in [2] and $P_{n, m}$ represents the states of a bidimensional Markov chain of $N_{k}$ elements $(k=1,2)$, $P_{1, j}$ and $P_{2, k}$ are the power assigned to users $j$ and $k$ of services 1 and 2 , respectively, $n_{1}$ and $n_{2}$ determine how many users of the $N_{1}$ and $N_{2}$ are active transmitting. Therefore the optimal power vector can be obtained by solving the linear $Q_{o S}$ equations in the powers. Since we are considering a single cell scenario, we will drop the subindices with respect to cells and redefine them to consider the single cell scenario with two-classes of traffic. In this way the Energy-bit to Noise ratio $\left(E_{b} / N_{0}\right)$ is established as

$$
\gamma_{i, j}=\frac{W}{R_{i, j}} \frac{P_{i, j} G_{i, j}}{\eta_{0} W+\sum_{\hat{\imath}=0} \sum_{\hat{\jmath}=0} P_{\hat{\imath}, \hat{\jmath}} G_{\hat{\imath}, \hat{\jmath}}}
$$

where $\gamma_{i, j}$ is the $\left(E b / N_{0}\right)_{\text {Target }}$ of service $i$ for user $j, P_{i, j}$ the power transmitted by user $j$ of service $i, G_{i, j}$, the channel gain of user $j$ of service $i, R_{i, j}$ the bit rate of user $j$ and service $i$ and $\hat{\imath}, \hat{\jmath}$, represents the rest of users who are transmitting and in consequence, interfering with $i, j$. In the case of $G_{\hat{\imath}, \hat{\jmath}}$ and $P_{\hat{\imath}, \hat{\jmath}}$, these are channel gain and power of the users interfering in the system to the uplink transmission of user $j$ of service $i$. 


\subsection{Algorithm Description}

Hence, in order to show how the algorithm works, or how we get results from the equations, the next procedure can help us to have a better understanding of the functioning of this algorithm. Figure 1]a shows a block diagram of the procedure to follow during the execution of the algorithm for evaluation purposes.

First, we have to define the activity parameters of the Markov chain $(\alpha, \beta$, $\delta$ and $\lambda$, see Figure 1, b), number of users, $N_{1}$ and $N_{2}$, in the system, also the power received in the uplink in the Base Station (BS) -this is in order to obtain channel gains and the necessary power from it-, bandwidth $W$, rates $R_{1}$ and $R_{2}$ for each service, and the noise density $\eta_{0}$.

Once defining these variables, we obtain by generating random numbers, the position of each of the $N_{1}+N_{2}$ users. This provides a random distance from the BS, and then we obtain $P_{i, j}$ and $G_{i, j}$, for each user in the system, independently of the service they require. This is done since users are located within the cell.

So, with this parameter we evaluate Equation (2) in order to obtain $\gamma_{\text {Target }}$ for both services.

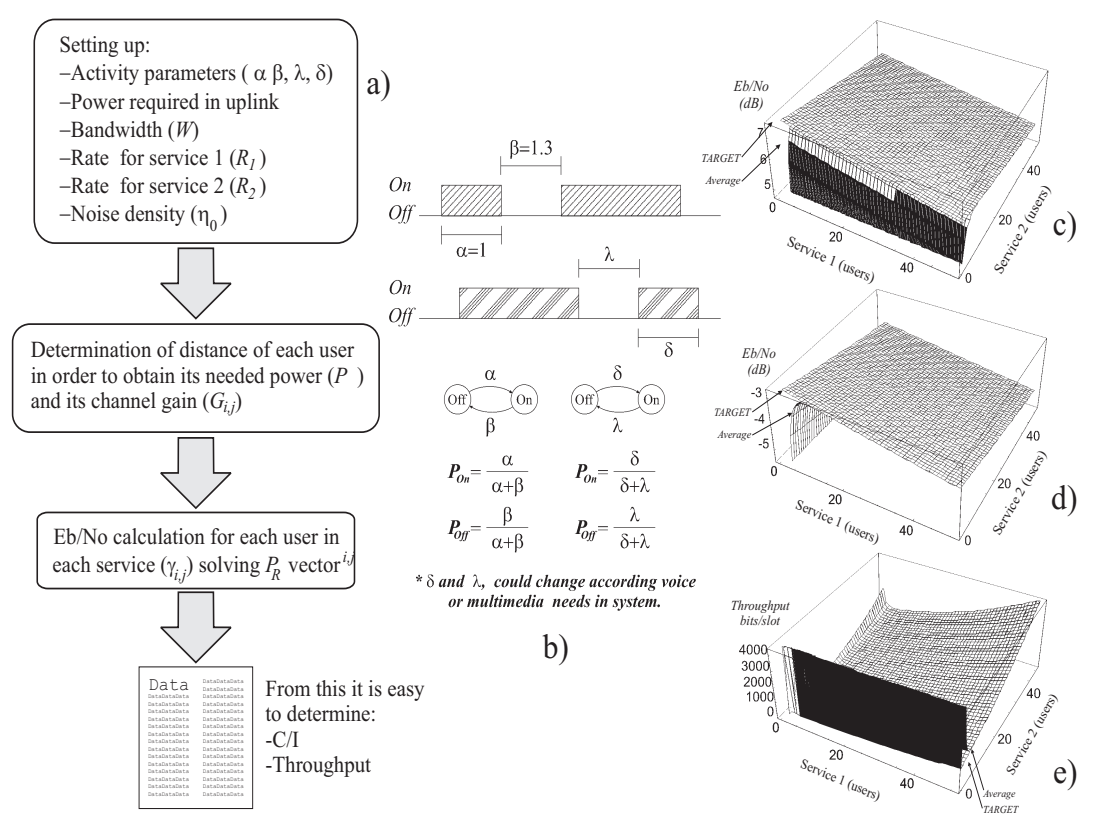

Fig. 1. a) Block diagram of the proposed algorithm, b) activity parameters of the Markov chain, c) and d) are the behavior of the system seen from service 1 and 2 respectively in response to the arriving of users, and e) is the throughput average and target of the system according to the arriving of users 


\section{$3 \quad$ Numerical Results}

Two services in the system are established, voice and multimedia data with a rate of $12.2 \mathrm{kbps}$ and $128 \mathrm{kbps}$ respectively. The activity parameters $\alpha$ and $\beta$ (that rule the Markov chain states, see Figure 1.b) were set in 1 second, $\delta$ and $\lambda$ were fixed with 0.5 and 0.65 seconds respectively. The diameter of the cell was established as $100 \mathrm{~m}$. Evaluation parameters accomplish with the WCDMA standard [34. In figures 1.c and1]d are shown the system behavior according to the arriving of users in transmission process in comparison with perfect power control. Notice that independently of the fading due the demand of the users, all of them manifest the same data rate asked or assigned since the beginning. In addition, because the $E_{b} / N_{0}$ does not manifest abrupt changes, the Carrier to Interference ratio $(C / I)$ obtained from it let us to get a constant throughput through the arriving of transmitting users, see Figure 1.e. This last figure is also compared with perfect power control.

\section{Conclusions and Future Work}

The power vector $P_{R}$ proposed in 2] was optimized using the Eng-Set distribution modeled by a bi-dimensional Markov chain for the two services proposed. But in addition, the $P_{R}$ can be enhanced for more data rate services, each dimension of the Markov chain system means another service.

Since $C / I$ does not affect the throughput behavior, the $Q \circ S$ required was satisfied and shows how the algorithm works properly independently of the arriving of users into the system. It is relevant to mention that the proposed algorithm manifests an equal or better behavior than a perfect power control.

However, in order to expand this work to general applications, it will be necessary add-on the multi cell capability in the algorithm, and in consequence take into account the inter-cell interference. Other point could be the use of multi rate with the purpose of maintain a higher $E_{b} / N_{0}$.

\section{References}

1. Novakovic Dejan M., Dukic Miroslav L., "Evolution of the Power Control Techniques for DS-CDMA Toward 3G Wireless Communication Systems", IEEE Communications Surveys, Fourth Quarter 2000.

2. Carrasco Loren, Femenias Guillem, "W-CDMA MAC Protocol for Multimedia Traffic Support", IEEE Vehicular Technology Conference Proceedings, VTC 2000-Spring Tokyo, Vol. 3, pp. 2193-2197, 2000.

3. Rappaport, Theodore S., Wireless Communications: Principles $\&$ Practice, Prentice Hall Inc., 2002.

4. Laiho Jaana, Wacker Achim, Novosad Tomás, Radio Network Planning and Optimisation for UMTS, John Wiley \& sons, LTD., 2002. 